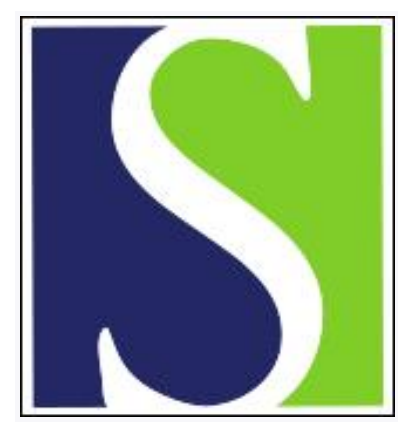

Scand J Work Environ Health 2005;31(2):97-107

https://doi.org/10.5271/sjweh.856

Issue date: Apr 2005

National job-exposure matrix in analyses of census-based estimates of occupational cancer risk

by Pukkala E, Guo J, Kyyrönen P, Lindbohm M-L, Sallmén M, Kauppinen $T$

Affiliation: Finnish Cancer Registry, Liisankatu 21 B, 00170 Helsinki, Finland. eero.pukkala@cancer.fi

The following articles refer to this text: 2008;34(6):444-450;

2020;46(3):268-277; 2022;48(7):540-548

Key terms: asbestos; census-based estimate; crystalline silica; exposure metric; Finland; job-exposure matrix; methodology; occupational cancer risk; prostate cancer; smoking

This article in PubMed: www.ncbi.nlm.nih.gov/pubmed/15864903 


\title{
National job-exposure matrix in analyses of census-based estimates of occupational cancer risk
}

\author{
by Eero Pukkala, PhD, ${ }^{1}$ Johannes Guo, MD, ${ }^{2}$ Pentti Kyyrönen, ${ }^{1}$ Marja-Liisa Lindbohm, DPH, ${ }^{2}$ Markku \\ Sallmén, PhD, ${ }^{2}$ Timo Kauppinen, $P h D^{2}$
}

\begin{abstract}
Pukkala E, Guo J, Kyyrönen P, Lindbohm M-L, Sallmén M, Kauppinen T. National job-exposure matrix in analyses of census-based estimates of occupational cancer risk. Scand J Work Environ Health 2005;31(2):97-107.
\end{abstract}

\begin{abstract}
Objectives The aim of this study was to increase the understanding of the alternative exposure metrics and analysis methods in studies applying job-exposure matrices in analyses of health outcomes, the association between crystalline silica and cancer being used as an example.

Methods Observed and expected numbers of cancer cases during 1971-1995 among Finns born in 1906-1945 were calculated for 393 occupational categories, as defined in the 1970 population census. According to the Finnish Cancer Registry, there were 43433 lung and 21444 prostate cancer cases. The Finnish job-exposure matrix (FINJEM) provided estimates of the proportion of exposed persons and the mean level of exposure among the exposed in each occupation.

Results The most comprehensive exposure metric included period- and age-specific estimates of exposure and an estimate of occupational stability, but also remarkably simpler metrics gave significantly elevated estimates of the risk ratio (RR) between 1.36 and 1.50 for lung cancer for occupations with the highest estimated cumulative silica exposure ( $\geq 10 \mathrm{mg} / \mathrm{m}^{3}$-years), allowing a lag time of 20 years. It proved important to adjust the risk ratios at least for the socioeconomic status and occupational exposure to asbestos. The risk ratios for prostate cancer were close to 1.0 in every model.

Conclusions The results showed that the FINJEM-based analysis was able to replicate the well-known association between exposure to crystalline silica and lung cancer. The FINJEM-based method gives valid results, and it can be used to analyze large sets of register-based data on health outcomes.
\end{abstract}

Key terms asbestos; crystalline silica; exposure metric; Finland; methodology; prostate cancer; smoking.

Risks of occupational cancer have been studied in several countries through analyses of the national cancer incidence or mortality data by occupational titles retrieved from a national census. Such studies have been carried out, for example, in Denmark (1), Finland (2), and the combined Nordic countries (3). Because of the large numbers of cases included, these studies work as a powerful tool for identifying occupations that have an elevated risk of contracting cancer. However, they suffer from one important disadvantage in that they are unable to identify directly causative agents or factors that would enable preventive measures against occupational cancer risks.

Job-exposure matrices were developed in the 1980s to overcome this defect of large register-based studies in which exposure assessment at the individual level was not feasible $(4,5)$. The matrices provided an easy way to analyze possibly causative exposures, but they also contained inaccurate estimates and could not take into account the variability of exposure between workers within an occupation. These features of the job-exposure matrix tend to attenuate observed risks (ie, to produce false negative findings). Job-exposure matrices may also reveal false positive findings because of multiple comparisons. Therefore, comprehensive matrices covering all occupations have not been particularly successful or popular among occupational epidemiologists, who have often preferred industry-specific cohorts and industry-specific exposure matrices because of their higher quality (6).

1 Finnish Cancer Registry, Institute for Statistical and Epidemiological Cancer Research, Helsinki, Finland.

2 Finnish Institute of Occupational Health, Helsinki, Finland.

Reprint requests to: Dr Eero Pukkala, Finnish Cancer Registry, Liisankatu 21 B, 00170 Helsinki, Finland. [E-mail: eero.pukkala@cancer.fi] 
The Finnish job-exposure matrix (FINJEM) was constructed in the 1990s for epidemiologic research, hazard surveillance, and risk assessment purposes (7). The aim was to approach the quality level of industryspecific matrices. Therefore, FINJEM was designed and constructed to produce quantitative exposure estimates, to address partly industry-specific occupations, and to take into account temporal changes in exposure. Exposure assessment was made by the best available experts within the Finnish Institute of Occupational Health. The estimates were based on exposure measurements as far as possible. They have also been carefully documented to increase their credibility. The quantitative nature of FINJEM and the availability of occupation-specific data on smoking and some other lifestyle factors were expected to enable the determination of occupational exposures and confounding factors more accurately than in previous similar studies.

The validity of an epidemiologic study also depends on the way the matrix is linked with health outcome data. The methods range from applying a simple constant exposure classification to each worker in the occupation to building up models that take into account historical changes in exposure patterns, an individual's age at exposure, duration of exposure, level of exposure, prevalence of exposure, lag time from exposure to outcome, occupational stability, and confounding lifestyle factors. The principal aim of this study was to assess the actual performance of alternative exposure metrics and analysis methods and to recommend a routine analysis procedure for future studies applying the FINJEM and other similar job-exposure matrices in the analysis of various health outcomes.

We selected the association between crystalline silica dust and lung cancer for the testing of different methodological alternatives. According to the evaluation of the International Agency for Research on Cancer (IARC) crystalline silica inhaled in the form of quartz or cristobalite from occupational sources is carcinogenic to humans (group 1) (8). Crystalline silica is still an important exposure in Finland with over 60000 exposed workers (3\% of the Finnish workforce). Lung cancer is considered causally associated with crystalline silica dust but further information of the exposure-response relationship is needed. We used prostate cancer to demonstrate that FINJEM-based analyses do not show an association with crystalline silica dust for a cancer when no association is expected to be observed.

\section{Study population and methods}

\section{Study population}

The study cohort consisted of all Finns born in 1906-1945 who participated in the national population census on 31
December 1970. The census files are maintained by Statistics Finland, and they are updated for death and emigration to allow exact person-year calculation. Data on the occupation and industry held for the longest time in 1970 were obtained from the 1970 population census records. The socioeconomic status (social class) for each person was determined based on his or her occupation, education, and industrial status in 1970 (9). In our analyses, farmers were included as a separate social class, and the rest of the population was classified as higher white-collar, clerical, skilled blue-collar, and unskilled workers.

\section{Cancer data}

The Finnish Cancer Registry has collected data on all incident cancer cases diagnosed in Finland since 1953. Physicians, hospitals, and pathological, cytological and hematological laboratories in the country are obliged to send notification of all cancer cases that come to their attention to the Registry. In addition, Statistics Finland annually sends the Registry a computerized file on death certificates in which a malignant disease is mentioned. The coverage of the Registry is virtually complete, and data accuracy is high (10).

For our study, all of the about 400000 cancers diagnosed in 1971-1995 among the 1.6 million persons born in 1906-1945 were extracted from the Finnish Cancer Register and sent to Statistics Finland for linkage with the 1970 census files. All persons residing in Finland since 1967 have been assigned a unique 11-digit personal identification number, which was used in the linkage. For $2.2 \%$ of the cancer cases, there was no record in the 1970 census file, and they were excluded from the cohort. Economically inactive persons were also excluded. In the remaining data, there were 30137 cases of lung cancer among the men and 3527 among the women. The number of prostate cancer cases was 18744 .

\section{Job-exposure matrix}

The occupational exposure estimates used in this study were based on FINJEM (7). The basic dimensions of exposure assessment in FINJEM are occupations, agents, and calendar periods. Each stratum is characterized by the proportion of exposed (P) and the mean level of exposure among the exposed (L). The exposure estimates are based on the judgment of about 20 experts at the Finnish Institute of Occupational Health. Estimates were generated for all agent-occupation combinations in which the proportion of exposed $(\mathrm{P})$ was suspected to have exceeded $5 \%$ any time after 1945 . The version of FINJEM used by us included 43 chemical 
exposures (table 1) and 311 occupational categories. To make the categories even more homogeneous in terms of their exposure pattern, 30 occupational categories were further divided into 2-9 industrial subcategories. The FINJEM periods used were 1945-1959 and 1960-1984.

The FINJEM database includes summary information on 8400 worktask-specific data sets on exposure measurements (over 80000 samples). Over 5000 samples of respirable quartz dust or quartz-containing dust from 1972-2000 were used by the industrial hygienists of the Finnish Institute of Occupational Health to assess silica exposure in about 40 exposed occupations. High validity was aimed at for the exposure estimates by using measurement data whenever available. Retrospective exposure estimates of silica in the FINJEM could not be directly validated because there were no other high-quality data to use for comparsion.

\section{Nonoccupational confounder data}

For the control of nonoccupational confounding factors, FINJEM contains estimates for smoking (proportion of daily smokers), alcohol consumption (average in grams/ week), dietary fat (proportion of regular butter users), and obesity [average body mass index (BMI)] by occupation. These data were obtained from the annual surveys carried out on the health behavior of the Finnish adult population by the Finnish National Public Health Institute during 1978-1991 (11). For rare occupations in which the number of respondents in the surveys was below 20, we estimated the values by using data of larger proxy occupations.

FINJEM measures occupational stability using two indices. The first is the annual probability of holding the same occupation as measured in censuses in 1970 and 1975 (12). The second is the average proportion of workyears spent in an occupation-out of the customary maximum time-calculated from the occupational histories of a case-control study of 2200 men (13).

\section{Exposure metric}

The exposure metric preferred in epidemiologic studies on chronic diseases such as cancer is usually cumulative exposure (CE). In this study, we calculated agentand occupation-specific cumulative-exposure estimates for every 5-year birth cohort (from 1906-1910 to 19411945) and 5-year calendar period of observation (from 1971-1975 to 1991-1995) (figure 1). Because occupational histories were not available apart from a crosssectional occupation in the 1970 census, the exposure for each birth cohort was assumed to start in the year when the average age of the birth cohort was 20 years and to end in the mid-year of the observation period or at 65 years of age, whichever came first. Because the actual work career in many occupations does not cover all 45 years between 20 and 65 years of age, the effect of correction for the occupational stability variables was tested. In each calendar year the annual average exposure was the product of the proportion of exposed (P) and the mean level of exposure among the exposed (L) in that occupation. If exposure took place before 1960, we used the FINJEM estimates for the period 1945-1959;

Table 1. Occupational exposures in the Finnish job-exposure matrix (FINJEM) and the default cut points for the cumulative exposure categories.

\begin{tabular}{|c|c|c|}
\hline \multirow[t]{2}{*}{ Agent } & \multicolumn{2}{|c|}{ Cut points } \\
\hline & Lower & Higher \\
\hline Formaldehyde (ppm-years) & 1 & 5 \\
\hline Organic solvents (score-years) & 20 & 200 \\
\hline $\begin{array}{l}\text { Aliphatic and alicyclic hydrocarbon } \\
\text { solvents (ppm-years) }\end{array}$ & 50 & 500 \\
\hline Aromatic hydrocarbon solvents (ppm-years) & 20 & 200 \\
\hline Chlorinated hydrocarbon solvents (ppm-years) & 5 & 50 \\
\hline Other organic solvents (ppm-years) & 50 & 500 \\
\hline Organic dust (score-years) & 10 & 100 \\
\hline Wood dust (mg/m³-years) & 5 & 50 \\
\hline Hardwood dust (mg/m³-years) & 2 & 20 \\
\hline Softwood dust (mg/m³-years) & 2 & 20 \\
\hline Pulp or paper dust (mg/m³-years) & 10 & 20 \\
\hline Flour dust (mg/m³-years) & 5 & 50 \\
\hline Plant dust (mg/m³-years) & 10 & 50 \\
\hline Textile dust (mg/m³-years) & 5 & 20 \\
\hline Leather dust (mg/m³-years) & 5 & 20 \\
\hline Animal dust (mg/m³-years) & 0.5 & 2 \\
\hline Synthetic polymer dust (mg/m³-years) & 5 & 20 \\
\hline Petroleum-based products (score-years) & 100 & 500 \\
\hline Gasoline (ppm-years) & 1 & 5 \\
\hline Oil mist (mg/m³-years) & 2 & 20 \\
\hline Bitumen fumes (mg/m³-years) & 2 & 10 \\
\hline Inorganic mineral dust (score-years) & 100 & 500 \\
\hline Asbestos ${ }^{a}\left(\mathrm{f} / \mathrm{cm}^{3}\right.$-years $)$ & 2 & 10 \\
\hline Manmade mineral fibers (MMMF) (f/cm³-years) & 2 & 5 \\
\hline Quartz dust (crystalline silica) a (mg/m³-years) & 1 & 10 \\
\hline Other mineral dusts (mg/m³-years) & 50 & 100 \\
\hline Metals (score-years) & 100 & 500 \\
\hline Iron (mg/m³-years) & 10 & 50 \\
\hline Lead ( $\mu \mathrm{mol} / /$-years) & 10 & 50 \\
\hline Chromium ( $\mu \mathrm{g} / \mathrm{m}^{3}$-years) & 50 & 500 \\
\hline Nickel ( $\mu \mathrm{g} / \mathrm{m}^{3}$-years) & 20 & 100 \\
\hline Cadmium ( $\mu \mathrm{g} / \mathrm{m}^{3}$-years) & 2 & 20 \\
\hline Arsenic ( $\mu \mathrm{g} / \mathrm{m}^{3}$-years) & 2 & 20 \\
\hline Carbon monoxide (ppm-years) & 200 & 500 \\
\hline Engine exhaust (score-years) & 100 & 500 \\
\hline Diesel exhaust (mg/m³-years) & 2 & 10 \\
\hline Gasoline engine exhaust (mg/m³-years) & 100 & 500 \\
\hline $\begin{array}{l}\text { Polycyclic aromatic hydro- } \\
\text { carbons (PAH) ( } \mu \mathrm{g} / \mathrm{m}^{3} \text {-years) }\end{array}$ & 10 & 50 \\
\hline Benzo(a)pyrene ( $\mu \mathrm{g} / \mathrm{m}^{3}$-years) & 1 & 5 \\
\hline Pesticides (score-years) & 100 & 500 \\
\hline Herbicides (mg/m³-years) & 0.005 & 0.02 \\
\hline Fungicides (mg/m³-years) & 0.02 & 0.2 \\
\hline Insecticides (mg/m³-years) & 0.002 & 0.1 \\
\hline
\end{tabular}

${ }^{a}$ Chemical agents adopted in our study. 


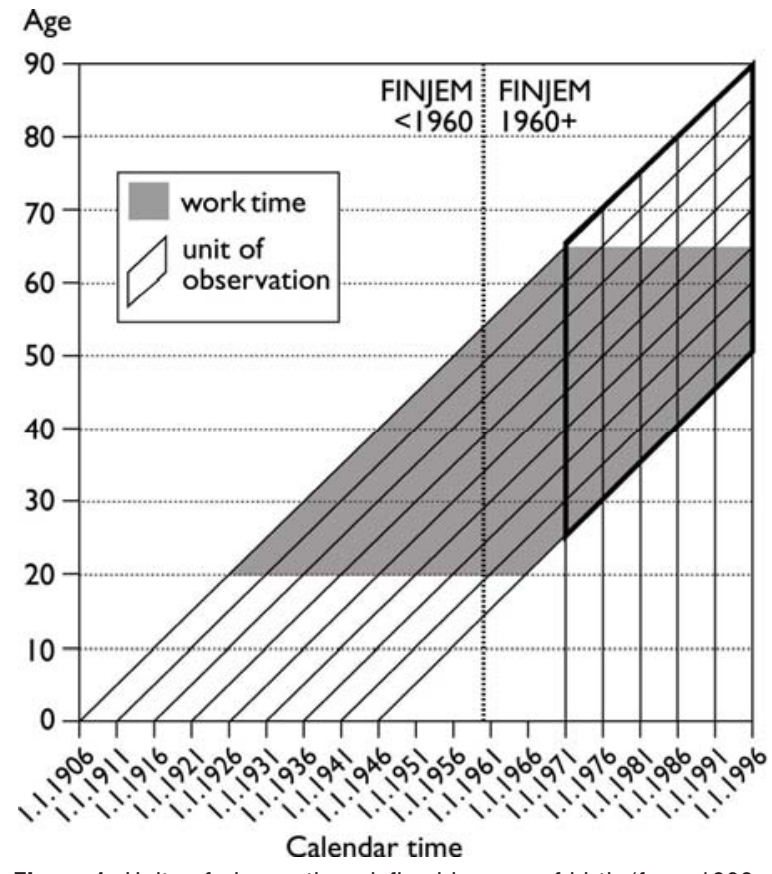

Figure 1. Units of observation, defined by year of birth (from 19061910 to 1941-1945) and calendar period of follow-up (from 19711975 to 1991-1995), theoretical work (exposure) time, and FINJEM periods used for the exposure estimation.

otherwise the estimates for the period 1960-1984 were used.

The lag period $\Phi$ was incorporated into the cumulative exposure by omitting exposure during $\Phi$ last years prior to the mid-year of the observation period. Most of the analyses were based on the traditional 20-year lag (eg, when cancer risk in 1971-1975 was studied, only exposures until 1953 were taken into account). We also calculated the profile likelihood of the data to optimize the length of the lag time in this data set. Profile likelihood is defined as $\mathrm{L}\left[\theta_{0}, \Phi\left(\theta_{0}\right)\right]$, where $\theta_{0}$ is to the maximum likelihood estimate (MLE) of exposure parameters $\theta$ with lag $\Phi$ as a nuisance parameter (14).

The exposure class limits for all chemical agents in the FINJEM were set a priori so that the highest cumulative exposure class was rather small and included mostly long-term workers with high exposure. The rest of the potentially exposed persons were divided into "medium" and "low" classes (table 1). The classes of cumulative exposure to crystalline silica were $<0.9 \mathrm{mg}$ / $\mathrm{m}^{3}$-years ("low"), $1.0-9.9 \mathrm{mg} / \mathrm{m}^{3}$-years ("medium") and $\geq 10 \mathrm{mg} / \mathrm{m}^{3}$-years ("high"), measured as respirable quartz dust.

\section{Statistical analyses}

For every occupation, the observed and expected numbers of cases were calculated for each gender, 5-year birth cohort, and 5-year calendar period. The expected number in each stratum was calculated by multiplying the number of person-years in that occupation by the cancer incidence rate of the entire Finnish population in the respective stratum. The standardized incidence ratio (SIR) used for this external comparison was defined as the ratio of observed to expected numbers of cases. These ratios were also calculated with adjustment for social class by splitting each stratum into five social classes and using national reference rates specific for social classes. The occupation-specific observed and expected numbers of cases were also combined according to the cumulative exposure to silica dust, and the standardized incidence ratios were calculated for each exposure category.

A Poisson regression analysis of the stratum-specific observed numbers of cases and person-years at risk was used to study the exposure-response patterns. In this internal comparison, the unexposed group was used as the reference category. The $95 \%$ confidence interval $(95 \% \mathrm{CI})$ was calculated for the risk ratio (RR). The effects of several parameters of the exposure metric and cofactors were studied to find an optimal strategy for the analyses. The most prevalent and significant occupational and nonoccupational lung cancer carcinogens among Finnish men (asbestos and smoking) were incorporated into the models.

\section{Results}

\section{Lung cancer}

Over $18 \%$ of the 30137 lung cancer cases among men were in occupations potentially entailing exposure to silica dust. The highest statistically significant occupation-specific standardized incidence ratios for the silica-exposed occupations were found for various occupations in the mining industry (table 2 ). The ratios adjusted for social class increased from 0.97 (95\% CI $0.96-0.99)$ for the unexposed category to $1.10(95 \% \mathrm{CI}$ $1.06-1.14), 1.17$ (95\% CI 1.12-1.21), and 1.31 (95\% CI $1.10-1.55)$ for the categories with a cumulative exposure of $\leq 0.9,1.0-9.9$, and $\geq 10 \mathrm{mg} / \mathrm{m}^{3}$-years, respectively. The respective ratios without social class adjustment were 0.93 (95\% CI $0.92-0.95$ ), 1.37 (95\% CI 1.32 1.42), 1.60 (95\% CI 1.53-1.66), and 1.60 (95\% CI $1.33-$ 1.90).

For the women the prevalence of potential exposure to silica dust was $4 \%$. Because the highest exposure class ( $\geq 10 \mathrm{mg} / \mathrm{m}^{3}$-years) included only five cases, it was merged with the medium exposure class (CE 1.0-9.9). The standardized incidence ratio adjusted for social class was 0.99 (95\% CI 0.96-1.03) for the unexposed, 1.03 (95\% CI 0.72-1.42) for those with low exposure, 
Table 2. Occupations exposed to crystalline silica dust according to the Finnish job exposure matrix (FINJEM), the levels (L) of silica and asbestos exposure, the proportions $(P)$ of exposed persons in two periods, and occupational stability. $(0=$ observed number of lung cancer cases among the men, SIR = standardized incidence ratio, $95 \% \mathrm{CI}=95 \%$ confidence interval)

\begin{tabular}{|c|c|c|c|c|c|c|c|c|c|c|c|c|}
\hline \multirow[t]{3}{*}{ Code $^{\mathrm{a}}$} & \multirow[t]{3}{*}{ Occupational title or industry } & \multicolumn{4}{|c|}{$\begin{array}{l}\text { Crystalline } \\
\text { silica exposure }\end{array}$} & \multirow{2}{*}{\multicolumn{2}{|c|}{$\begin{array}{c}\text { Asbestos } \\
\text { exposure } \\
\text { mean } \\
1945-1984\end{array}$}} & \multirow{3}{*}{$\begin{array}{c}\text { Annual } \\
\text { occupa- } \\
\text { tional } \\
\text { stability } \\
\text { in } \\
1970- \\
1975 \\
(\%)\end{array}$} & \multirow{3}{*}{$\begin{array}{c}\text { Work- } \\
\text { years } \\
\text { in this } \\
\text { occu- } \\
\text { pation } \\
\text { (\% } \\
\text { of all } \\
\text { years) }\end{array}$} & \multicolumn{3}{|c|}{$\begin{array}{l}\text { Lung cancer incidence } \\
\text { 1971-1995 }\end{array}$} \\
\hline & & \multicolumn{2}{|c|}{$1945-1959$} & \multicolumn{2}{|c|}{$1960-1984$} & & & & & 0 & SIR & $95 \% \mathrm{Cl}$ \\
\hline & & $\underset{\left(\mathrm{mg} / \mathrm{m}^{3}\right)}{\mathrm{L}}$ & $\begin{array}{c}P \\
(\%)\end{array}$ & $\stackrel{\mathrm{L}}{\mathrm{L}}\left(\mathrm{mg} / \mathrm{m}^{3}\right)$ & $\begin{array}{c}P \\
(\%)\end{array}$ & $\underset{\left(\mathrm{f} / \mathrm{cm}^{3}\right)}{\mathrm{L}}$ & $\begin{array}{c}P \\
(\%)\end{array}$ & & & & & \\
\hline 019 & Laboratory assistant & 0.02 & 5 & 0.01 & 6 & . & . & 87 & 88 & 23 & 0.99 & $0.63-1.49$ \\
\hline 400.1 & Miner, quarry worker, metal ore & 0.50 & 100 & 0.10 & 100 & 0.7 & 10 & 77 & 51 & 36 & 3.26 & $2.28-4.51$ \\
\hline 400.9 & Miner, quarry worker, except metal ore & 0.15 & 100 & 0.12 & 100 & 2.8 & 41 & 77 & 51 & 181 & 1.85 & $1.59-2.14$ \\
\hline 410 & Deed-drilling-machine operator & 0.03 & 100 & 0.03 & 100 & . & . & 70 & 51 & 31 & 1.98 & $1.34-2.81$ \\
\hline 420 & Concentration plant worker & 0.03 & 40 & 0.03 & 40 & 14 & 3 & 75 & 51 & 15 & 1.16 & $0.65-1.92$ \\
\hline 490 & $\begin{array}{l}\text { Mining, quarry worker, not elsewhere } \\
\text { classified }\end{array}$ & 0.40 & 90 & 0.14 & 90 & 0.2 & 19 & 68 & 51 & 70 & 1.73 & $1.35-2.19$ \\
\hline 630.1 & Smelter worker, iron or steel & 0.20 & 100 & 0.16 & 100 & . & . & 78 & 81 & 28 & 1.04 & $0.69-1.50$ \\
\hline 630.2 & Smelter worker, nonferrous metals & 0.15 & 100 & 0.12 & 100 & . & . & 78 & 81 & 17 & 1.13 & $0.66-1.81$ \\
\hline 630.9 & Smelter workers, not elsewhere classified & 0.15 & 100 & 0.12 & 100 & . & . & 78 & 81 & 14 & 0.85 & $0.46-1.43$ \\
\hline 635.1 & Foundry worker, iron or steel & 0.25 & 100 & 0.20 & 100 & . & . & 78 & 68 & 43 & 1.27 & $0.92-1.70$ \\
\hline 635.2 & Foundry worker, nonferrous metals & 0.20 & 100 & 0.15 & 100 & . & . & 78 & 68 & 5 & 0.89 & $0.29-2.09$ \\
\hline 635.9 & Foundry worker, not elsewhere classified & 0.40 & 100 & 0.18 & 100 & . & . & 78 & 68 & 73 & 1.25 & $0.98-1.57$ \\
\hline 639.1 & $\begin{array}{l}\text { Metal mill workers, not elsewhere classified, } \\
\text { iron or steel }\end{array}$ & 0.20 & 50 & 0.15 & 55 & . & . & 60 & 72 & 24 & 1.33 & $0.85-1.97$ \\
\hline 639.2 & $\begin{array}{l}\text { Metal mill worker, not elsewhere classified, } \\
\text { nonferrous metals }\end{array}$ & 0.15 & 60 & 0.10 & 59 & . & $\cdot$ & 60 & 72 & 2 & 0.63 & $0.08-2.29$ \\
\hline 639.9 & Metal mill worker, not elsewhere classified & 0.15 & 35 & 0.10 & 36 & . & . & 60 & 72 & 42 & 1.15 & $0.83-1.56$ \\
\hline 652.9 & Machine repairer, except cars & 0.05 & 40 & 0.03 & 36 & . & . & 83 & 68 & 305 & 1.00 & $0.89-1.12$ \\
\hline 661 & Electric machine operator & 0.02 & 30 & 0.01 & 30 & . & . & 68 & 72 & 53 & 0.92 & $0.69-1.20$ \\
\hline 673 & Construction carpenter & 0.02 & 75 & 0.02 & 76 & 0.5 & 70 & 89 & 54 & 1885 & 1.19 & $1.14-1.25$ \\
\hline 690 & Bricklayer, tile setter & 0.10 & 100 & 0.10 & 100 & 0.3 & 51 & 89 & 52 & 336 & 1.32 & $1.19-1.47$ \\
\hline 691 & Construction element worker & 0.03 & 100 & 0.03 & 100 & . & . & 67 & 59 & 9 & 1.65 & $0.75-3.13$ \\
\hline 693 & Concrete or cement shutterer & 0.10 & 90 & 0.08 & 90 & . & . & 77 & 59 & 202 & 1.26 & $1.09-1.45$ \\
\hline 697 & Building hand & 0.20 & 100 & 0.10 & 100 & 1.8 & 69 & 73 & 46 & 1144 & 1.09 & $1.02-1.15$ \\
\hline 698.2 & Assisting workers, road building & 0.02 & 100 & 0.03 & 100 & . & . & 68 & 59 & 503 & 1.09 & $1.00-1.19$ \\
\hline 698.3 & $\begin{array}{l}\text { Assisting building worker, not elsewhere } \\
\text { classified }\end{array}$ & 0.02 & 100 & 0.03 & 100 & . & . & 68 & 59 & 140 & 1.03 & $0.97-1.22$ \\
\hline 699 & $\begin{array}{l}\text { Construction workers, not elsewhere } \\
\text { classified }\end{array}$ & 0.08 & 70 & 0.06 & 70 & 1.1 & 16 & 66 & 59 & 101 & 1.29 & $1.05-1.57$ \\
\hline 710 & Glass former & 0.60 & 80 & 0.44 & 80 & . & . & 81 & 53 & 26 & 1.01 & $0.66-1.48$ \\
\hline 711 & Potter & 0.50 & 100 & 0.40 & 90 & . & . & 71 & 53 & 25 & 1.01 & $0.65-1.49$ \\
\hline 712 & Glass or ceramics kiln operator & 0.40 & 70 & 0.30 & 70 & . & . & 76 & 53 & 15 & 1.21 & $0.67-1.99$ \\
\hline 713 & Glass or ceramics decorator & 0.15 & 50 & 0.10 & 50 & . & . & 72 & 53 & 10 & 1.56 & $0.75-2.87$ \\
\hline 714 & Glass or clay mixer & 0.20 & 100 & 0.15 & 70 & . & . & 62 & 53 & 5 & 1.97 & $0.64-4.59$ \\
\hline 719 & $\begin{array}{l}\text { Glassor ceramics workers, not elsewhere } \\
\text { classified }\end{array}$ & 0.30 & 95 & 0.20 & 95 & . & . & 73 & 53 & 8 & 0.80 & $0.35-1.58$ \\
\hline 739.2 & Paint or pharmaceutical maker & 0.10 & 30 & 0.06 & 34 & 0.01 & 10 & 75 & 69 & 14 & 0.75 & $0.41-1.26$ \\
\hline 739.9 & Chemical worker, not elsewhere classified & 0.15 & 15 & 0.13 & 16 & 0.3 & 4 & 75 & 69 & 6 & 0.90 & $0.33-1.96$ \\
\hline 752.2 & Plastic product worker & 0.08 & 10 & 0.05 & 16 & . & . & 76 & 38 & 31 & 0.94 & $0.64-1.34$ \\
\hline 752.9 & $\begin{array}{l}\text { Plastic product worker, not elsewhere } \\
\text { classified }\end{array}$ & 0.08 & 15 & 0.05 & 25 & . & . & 76 & 38 & 15 & 1.16 & $0.65-1.92$ \\
\hline 756 & Stone cutter & 0.60 & 90 & 0.44 & 87 & . & . & 79 & 44 & 39 & 1.26 & $0.89-1.72$ \\
\hline 758 & Concrete product or element worker & 0.15 & 100 & 0.14 & 100 & . & . & 69 & 44 & 41 & 1.10 & $0.79-1.50$ \\
\hline 831.5 & Cleaning worker, mineral product industry & 0.10 & 100 & 0.06 & 100 & . & . & 85 & 27 & 1 & 1.36 & $0.03-7.60$ \\
\hline 831.7 & Cleaning worker, building & 0.30 & 100 & 0.14 & 100 & . & . & 85 & 27 & 2 & 5.77 & $0.70-20.8$ \\
\hline
\end{tabular}

a The fourth digit indicated that, in the FINJEM, the official census occupation was divided into subcategories according to industry.

and 1.48 (95\% CI 1.19-1.82) for those with mediumhigh exposure. The respective values not adjusted for social class were 0.99 (95\% CI 0.95-1.02), 1.20 (95\% CI 0.84-1.66), and 1.78 (95\% CI 1.43-2.19). Because the number of cases exposed to silica dust among the women was small, we restricted further analyses (by Poisson regression) to the men.

The effects of different adjustment factors on the risk ratio of lung cancer, calculated for various categories of cumulative exposure and two different lengths of lag 
Table 3. Effect of lag time and alternative adjustment factors on the relative risk (RR) of lung cancer by cumulative exposure to crystalline silica dust among Finnish men in 1971-1995 (0 = observed number of cases; $95 \% \mathrm{Cl}=95 \%$ confidence interval). Reference category: no exposure to crystalline silica dust (24 591 observed cases for lag time of 0 years; 24598 cases for lag time of 20 years).

\begin{tabular}{|c|c|c|c|c|c|c|c|c|c|}
\hline \multirow[t]{3}{*}{ Lag time (adjustment factors included in the model) } & \multicolumn{9}{|c|}{ Cumulative exposure to crystalline silica dust ( $\mathrm{mg} / \mathrm{m}^{3}$-years) } \\
\hline & \multicolumn{3}{|c|}{$\leq 0.9$} & \multicolumn{3}{|c|}{$1.0-9.9$} & \multicolumn{3}{|c|}{$\geq 10$} \\
\hline & 0 & $\mathrm{RR}$ & $95 \% \mathrm{Cl}$ & 0 & $\mathrm{RR}$ & $95 \% \mathrm{Cl}$ & 0 & RR & $95 \% \mathrm{Cl}$ \\
\hline \multicolumn{10}{|l|}{ No lag time } \\
\hline Age, period & 2999 & 1.48 & $1.42-1.53$ & 2339 & 1.75 & $1.68-1.83$ & 208 & 1.46 & $1.28-1.68$ \\
\hline Age, period, social class & 2999 & 1.18 & $1.13-1.23$ & 2339 & 1.31 & $1.25-1.37$ & 208 & 1.22 & $1.06-1.40$ \\
\hline Age, period, smoking & 2999 & 1.23 & $1.18-1.28$ & 2339 & 1.19 & $1.14-1.25$ & 208 & 1.26 & $1.10-1.44$ \\
\hline Age, period, asbestos & 2999 & 1.29 & $1.23-1.36$ & 2339 & 1.50 & $1.42-1.59$ & 208 & 1.44 & $1.26-1.65$ \\
\hline Age, period, social class, smoking & 2999 & 1.17 & $1.12-1.21$ & 2339 & 1.14 & $1.08-1.19$ & 208 & 1.20 & $1.05-1.38$ \\
\hline Age, period, social class, asbestos & 2999 & 1.09 & $1.03-1.14$ & 2339 & 1.18 & $1.12-1.26$ & 208 & 1.23 & $1.07-1.41$ \\
\hline Age, period, smoking, asbestos & 2999 & 1.09 & $1.04-1.15$ & 2339 & 1.02 & $0.96-1.09$ & 208 & 1.24 & $1.08-1.43$ \\
\hline Age, period, social class, smoking, asbestos & 2999 & 1.05 & $0.99-1.10$ & 2339 & 0.99 & $0.93-1.05$ & 208 & 1.20 & $1.05-1.38$ \\
\hline \multicolumn{10}{|l|}{ Lag time of 20 years } \\
\hline Age, period & 3115 & 1.48 & $1.43-1.54$ & 2277 & 1.73 & $1.66-1.80$ & 129 & 1.69 & $1.42-2.01$ \\
\hline Age, period, social class & 3115 & 1.18 & $1.13-1.23$ & 2277 & 1.29 & $1.23-1.35$ & 129 & 1.42 & $1.20-1.69$ \\
\hline Age, period, smoking & 3115 & 1.22 & $1.18-1.27$ & 2277 & 1.18 & $1.12-1.24$ & 129 & 1.47 & $1.24-1.75$ \\
\hline Age, period, asbestos & 3115 & 1.34 & $1.27-1.40$ & 2277 & 1.39 & $1.31-1.48$ & 129 & 1.68 & $1.41-2.00$ \\
\hline Age, period, social class, smoking & 3115 & 1.16 & $1.11-1.21$ & 2277 & 1.13 & $1.07-1.18$ & 129 & 1.42 & $1.19-1.69$ \\
\hline Age, period, social class, asbestos & 3115 & 1.10 & $1.05-1.16$ & 2277 & 1.14 & $1.07-1.21$ & 129 & 1.43 & $1.20-1.70$ \\
\hline Age, period, smoking, asbestos & 3115 & 1.09 & $1.04-1.15$ & 2277 & 1.00 & $0.94-1.07$ & 129 & 1.46 & $1.23-1.74$ \\
\hline Age, period, social class, smoking, asbestos ["preferred model "] & 3115 & 1.05 & $1.00-1.10$ & 2277 & 0.97 & $0.91-1.03$ & 129 & 1.42 & $1.20-1.70$ \\
\hline $\begin{array}{l}\text { Age, period, asbestos; social classes 1-2 (white collar) } \\
\text { and farmers excluded }\end{array}$ & 3028 & 1.07 & $1.02-1.13$ & 2231 & 1.04 & $0.97-1.11$ & 122 & 1.48 & $1.24-1.77$ \\
\hline
\end{tabular}

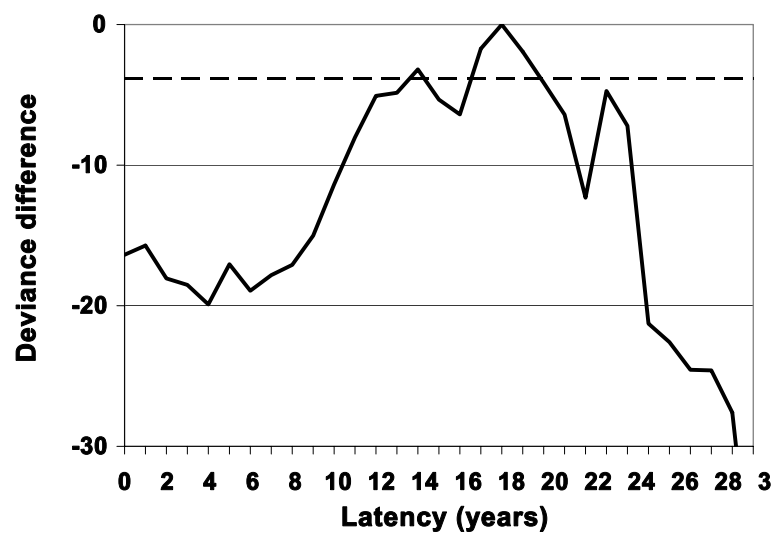

Figure 2. Difference $\left[-2 \times\left(L_{i}-L_{\max }\right)\right]$ between the maximum log likelihood ( $\mathrm{L}_{\max }$, reached at a lag time of 18 years) and the log likelihood $\mathrm{L}_{\mathrm{i}}(\mathrm{i}=0 \ldots 30$ years $)$ derived from a Poisson regression model of silica dust and lung cancer (FINJEM-based analysis). $L_{i}$ values below the dotted line differ significantly $(P<0.05)$ from the $L_{\max }$.

time ( 0 and 20 years) are presented in table 3 . The basic model, adjusted only for age and period of followup, showed significantly increased risk ratios between 1.46 and 1.75 , irrespective of the exposure category or lag time. In this setting, adjustment for age and period had no effect on the risk ratios (ie, the factors could be dropped from the model).

Adding the smoking variable strongly reduced the risk ratio, especially in the category of medium silica exposure (1.0-9.9 mg/m³ -years). Social class alone had a similar effect, indicating a high correlation between the prevalence of smoking and social class among
Finnish men. Including both smoking and social class in the same model produced risk ratio estimates that were similar to that of either of the two factors alone. The parameter correlation between social class and smoking was $0.4-0.8$, depending on the model used.

Occupational exposure to asbestos had an independent effect as a confounder. In a model including smoking and asbestos there was a significantly increased risk ratio in the category of high silica exposure $(\geq 10 \mathrm{mg} /$ $\mathrm{m}^{3}$-years), but very little or no excess in the low and medium exposure categories. In the most extensive model (including age and period of follow-up, social class, smoking, and asbestos exposure) the risk ratio in the highest silica exposure category increased from 1.20 to 1.42 when the lag time was increased from 0 to 20 years. This model was considered the most valid for studying lung cancer, and it is hereafter referred to as the "preferred model". In the preferred model, the maximum likelihood was reached with a lag time of 18 years, but the likelihood for lag times of 14,17 , or 19 20 years did not significantly differ from the maximum value (figure 2).

The risk coefficients for the co-factors included in the "preferred model " are shown in table 4. The risk ratio for lung cancer increased 1.24 -fold per each $10 \%$ unit of prevalence of smokers in the occupation. In addition, white-collar workers had a significantly lower basic risk level than the blue-collar workers or farmers. Asbestos showed a marked increasing risk effect already with cumulative exposure levels as low as 2 fibers $/ \mathrm{cm}^{3}$-years. 
In several earlier studies on occupational exposures, the effect of lifestyle confounding was controlled by restricting the analyses to only blue-collar workers, instead of adjusting for social class. We showed the effect of this method on our "preferred model " by excluding all white-collar workers (social classes 1-2) and farmers. Although the number of lung cancer cases decreased from 30137 to 16346 , this reduction occurred almost entirely in the large unexposed category. The risk ratios (and their 95\% CI) for the cumulative exposure categories for silica exposure were very similar to those obtained from the full data set (bottom line in table 3 ).

In the preceding exercise, we used a complex calculation algorithm to define the "preferred exposure metric". In other instances, fewer data on exposure may have been available or simpler calculation procedures may have been preferred to avoid massive data handling. We studied the practical impact of the reduction on the accuracy of the exposure metric in comparison with results with the "preferred exposure metric".

When we used the estimates referring to exposure conditions during 1960-1984 also for the period before 1960 (metric 2 in table 5), the risk ratio estimate for the highest exposure category decreased from 1.42 to 1.31 . Fewer people had high cumulative exposure because the exposure levels decreased in 32 out of the 39 silica-exposed occupations over time (table 2).

If we ignored the estimated duration of exposure and simply adopted the average proportion of exposed multiplied by the mean level of exposure from the FINJEM
Table 4. Relative risk (RR) coefficients for cofactors adjusted for each other, age, period, and cumulative exposure to crystalline silica dust among Finnish men in 1971-1995, lag time of 20 years. $(95 \% \mathrm{Cl}=95 \%$ confidence interval, $\mathrm{f}=$ fibers $)$

\begin{tabular}{|c|c|c|c|c|c|c|}
\hline \multirow[t]{2}{*}{ Variable } & \multicolumn{3}{|c|}{ Lung } & \multicolumn{3}{|c|}{ Prostate } \\
\hline & $\begin{array}{l}\text { Observ- } \\
\text { ed cases } \\
\text { (N) }\end{array}$ & $\begin{array}{l}-R R \\
s\end{array}$ & $95 \% \mathrm{Cl}$ & $\begin{array}{l}\text { Observ- } \\
\text { ed cases } \\
\text { (N) }\end{array}$ & - RR & $95 \% \mathrm{Cl}$ \\
\hline \multicolumn{7}{|l|}{ Social class } \\
\hline Higher white-collar & r 1314 & 0.60 & $0.55-0.65$ & 2045 & 1.24 & $1.13-1.37$ \\
\hline Clerical & 4355 & 0.79 & $0.75-0.84$ & 3470 & 1.10 & $1.01-1.19$ \\
\hline Skilled blue-collar & 13047 & 1.02 & $0.97-1.07$ & 6390 & 1.04 & $0.97-1.12$ \\
\hline Unskilled & 3299 & 1.00 & Reference & 1195 & 1.00 & Reference \\
\hline Farmer & 8122 & 1.11 & $1.04-1.18$ & 5644 & 0.95 & $0.87-1.04$ \\
\hline Smoking a & 30137 & 1.24 & $1.22-1.27$ & 18744 & 0.98 & $0.95-1.00$ \\
\hline \multicolumn{7}{|c|}{ Cumulative asbestos exposure } \\
\hline None & 24007 & 1.00 & Reference & 16089 & 1.00 & Reference \\
\hline$<2 \mathrm{f} / \mathrm{cm}^{3}$-years & 1893 & 1.08 & $1.02-1.13$ & 947 & 1.01 & $0.94-1.08$ \\
\hline $2-9.9 \mathrm{f} / \mathrm{cm}^{3}$-years & 2493 & 1.23 & $1.16-1.30$ & 968 & 1.02 & $0.93-1.11$ \\
\hline$\geq 10 \mathrm{f} / \mathrm{cm}^{3}$-years & 1744 & 1.26 & $1.17-1.35$ & 740 & 0.93 & $0.84-1.04$ \\
\hline
\end{tabular}

a Per $10 \%$ units in prevalence of smokers in occupation.

for the period 1960-1984 for all the participants, the trend pattern (metric 3 in table 5) was similar to that obtained from the "preferred metric". The class limits between the exposure categories cannot be kept the same for this metric (not including the time component) and cumulative exposure (including the time component).

Another way of simplifying the exposure metric is to drop the very low exposure categories, for which the

Table 5. Effect of simplification of the exposure metric on the relative risk (RR) estimate of lung cancer by cumulative exposure to crystalline silica dust among Finnish men in 1971-1995. . $^{0} 0$ observed number of cases; $95 \% \mathrm{Cl}=95 \%$ confidence interval, $\mathrm{P}=$ proportion of exposed persons in occupation, $\mathrm{L}=$ average exposure among the exposed persons)

\begin{tabular}{|c|c|c|c|c|c|c|c|c|c|c|c|c|c|c|c|c|}
\hline \multirow{3}{*}{$\begin{array}{l}\text { Met- } \\
\text { ric } \\
\text { num- } \\
\text { ber }\end{array}$} & \multicolumn{7}{|c|}{ Parameters included in the exposure metric } & \multicolumn{9}{|c|}{ Exposure to crystalline silica dust ${ }^{b}$} \\
\hline & \multirow{2}{*}{$\mathrm{P}$} & \multirow[t]{2}{*}{ L } & \multirow{2}{*}{$\begin{array}{l}\text { Cumu- } \\
\text { lati- } \\
\text { vity/ } \\
\text { age }\end{array}$} & \multirow{2}{*}{$\begin{array}{l}\text { Histo- } \\
\text { rical } \\
\text { FINJEM }\end{array}$} & \multirow{2}{*}{$\begin{array}{l}\text { Thres- } \\
\text { hold } \\
\text { value } \\
\text { of } L \\
\left(\mathrm{mg} / \mathrm{m}^{3}\right)\end{array}$} & \multirow{2}{*}{\multicolumn{2}{|c|}{$\begin{array}{l}\text { Occupa-Years } \\
\text { tional employ- } \\
\text { stabi- ed }{ }^{d} \\
\text { lityc }{ }^{c}\end{array}$}} & \multicolumn{3}{|c|}{ Low } & \multicolumn{3}{|c|}{ Medium } & \multicolumn{3}{|c|}{ High } \\
\hline & & & & & & & & 0 & $\mathrm{RR}$ & $95 \% \mathrm{Cl}$ & 0 & $\mathrm{RR}$ & $95 \% \mathrm{Cl}$ & 0 & $\mathrm{RR}$ & $95 \% \mathrm{Cl}$ \\
\hline $1^{e}$ & + & + & + & + & 0 & . & - & 3115 & 1.05 & $1.00-1.10$ & 2277 & 0.97 & $0.91-1.03$ & 129 & 1.42 & $1.20-1.70$ \\
\hline 2 & + & + & + & - & 0 & . & - & 3129 & 1.06 & $1.01-1.11$ & 2357 & 1.00 & $0.94-1.06$ & 35 & 1.31 & $0.94-1.83$ \\
\hline 3 & + & + & - & - & 0 & . & - & 3118 & 1.05 & $1.00-1.10$ & 2279 & 0.97 & $0.91-1.03$ & 128 & 1.45 & $1.22-1.72$ \\
\hline 4 & + & + & + & + & 0.1 & . & - & 235 & 1.00 & $0.87-1.14$ & 1717 & 0.89 & $0.83-0.96$ & 129 & 1.42 & $1.19-1.69$ \\
\hline $4 b$ & + & + & + & + & 0.2 & . & - & 6 & 0.96 & $0.43-2.15$ & 206 & 1.26 & $1.09-1.44$ & 118 & 1.38 & $1.15-1.66$ \\
\hline 5 & - & + & + & + & 0 & . & - & 3124 & 1.06 & $1.01-1.11$ & 2272 & 0.97 & $0.91-1.03$ & 125 & 1.36 & $1.14-1.63$ \\
\hline 6 & + & - & + & + & . & $\cdot$ & - & 3107 & 1.09 & $1.04-1.15$ & 2272 & 0.97 & $0.91-1.02$ & 142 & 0.94 & $0.79-1.12$ \\
\hline 7 & + & + & + & + & 0 & Low & - & 1216 & 1.01 & $0.95-1.07$ & 1943 & 0.88 & $0.82-0.92$ & 129 & 1.36 & $1.14-1.61$ \\
\hline 8 & + & + & + & + & 0 & $\mathrm{High}^{\mathrm{c}}$ & - & 1899 & 1.18 & $1.08-1.28$ & 334 & 1.31 & $1.15-1.49$ & - & . & . \\
\hline 9 & + & + & + & + & 0 & $\cdot$ & + & 3119 & 1.04 & $0.99-1.10$ & 2296 & 0.98 & $0.92-1.05$ & 106 & 1.50 & $1.23-1.82$ \\
\hline
\end{tabular}

a Reference category: no exposure to crystalline silica dust; lag time of 20 years; adjustment for age, calendar period of follow-up, social class, smoking, and asbestos exposure.

b Low-medium and medium-high cut points: metric 3: 0.052 and $0.40 \mathrm{mg} / \mathrm{m}^{3}$; metric 5: 1.5 and 10.2 mg/m³-years; metric 6: 22.5 and 40.0 "proportionyears"; all other models [ie, those with various cumulative exposure measures (proportion of exposed multiplied by average length of expsoure)]: 1.0 and $10 \mathrm{mg} / \mathrm{m}^{3}$-years.

c Categories below and above median occupational stability rate among all occupations.

d The cumulative exposure estimate included only the number of workyears that persons stay in that occupation on the average.

e "Preferred model "from table 3. 
actual exposure status of the participants is often uncertain. When we excluded all the occupations with exposure levels of $<0.1 \mathrm{mg} / \mathrm{m}^{3}$ (about one-third of the exposed occupations, depending on the exposure period), the number of observations in the lowest exposure category ( $\leq 0.9 \mathrm{mg} / \mathrm{m}^{3}$-years) dropped from 3115 to 235 , but nothing changed in the highest exposure category (metric 4 in table 5). Raising the threshold exposure to 0.2 $\mathrm{mg} / \mathrm{m}^{3}$ made the lowest exposure category practically disappear, but the medium category $\left(1.0-9.9 \mathrm{mg} / \mathrm{m}^{3}\right.$ years) showed a significant increase in risk (metric $4 \mathrm{~b}$ in table 5).

If we took into account only the average exposure levels (metric 5 in table 5), the trend pattern was still similar to that of the "preferred metric". However, including only the prevalence of exposure, instead, was inappropriate, showing no risk in the middle and high cumulative exposure classes, and a slightly decreasing exposure-response relationship (metric 6 in table 5).

When the analysis was stratified according to the annual occupational stability, the more stable fraction (index above the median) showed higher risk ratios than the less stable fraction in the low and medium exposure categories. There were no stable occupations with cumulative exposure $\geq 10 \mathrm{mg} / \mathrm{m}^{3}$-years (metrics $7-8$ in table 5). The alternative method with which to characterize occupational stability was to utilize information on the average proportion of time in each occupation during the work history. For example, a cleaning worker spends an average of only $27 \%$ of his or her worklife in this occupation (ie, only 12 years of the 45 years between 20 and 65 years of age), while the respective proportion for a laboratory assistant is $88 \%$ (table 2). In this exercise (metric 9 in table 5), we placed the estimated work period at the beginning of the work career, assuming that occupational exposure to silica dust starts at the age of 20 years. This approach resulted in risk ratios that were approximately similar to those obtained with the "preferred metric".

\section{Prostate cancer}

The risk ratio for prostate cancer in the highest silica exposure category ( $\geq 10 \mathrm{mg} / \mathrm{m}^{3}$-years) was below 1.0 in every model, and adopting a 20 -year lag did not affect the risk ratios. The incidence of prostate cancer varies according to social class (15), and therefore adjustment for social class was considered essential. Although the risk ratio tended to increase among the higher social classes also in this data set (table 4), the effect of social class adjustment on the risk ratio estimates for silica exposure remained small. Smoking and asbestos had only a negligible effect on the risk estimates, as expected (table 4).

In the model including age, period, and social class and adopting a 20-year lag, the risk ratio for the lowest silica exposure category $\left(0.1-0.9 \mathrm{mg} / \mathrm{m}^{3}\right.$-years $)$ was 0.98 (95\% CI $0.92-1.06,1348$ cases of prostate cancer) in comparison with the unexposed category (16 474 cases). For the intermediate exposure category of 1.0-9.9 $\mathrm{mg} / \mathrm{m}^{3}$-years the risk ratio was 1.04 (95\% CI 0.96-1.12, 860 cases $)$, and for the highest category $\left(\geq 10 \mathrm{mg} / \mathrm{m}^{3}\right.$ years) it was 0.87 (95\% CI 0.68-1.12, 62 cases).

\section{Discussion}

\section{Methodological issues}

Our results demonstrated that the FINJEM-based analysis was able to replicate the well-known association between crystalline silica dust exposure and lung cancer. No association was found for prostate cancer. These results were as expected prior to the study. We also had reasonable results for the cofactors included in the analyses. These findings strengthen our belief that the FINJEM-based method can be used to analyze large register-based data sets, such as that used in this analysis. One prerequisite for this use was that we were able to use about two person-years of work to construct the FINJEM. Its occupation-specific risk estimates-both proportions and average exposure levels - are based on the expertise of many specialists of occupational hygiene and a large number of exposure measurements (7). There are ongoing processes to copy or modify FINJEM for similar risk estimation in the other Nordic countries and also several other countries.

On the basis of experiences from earlier studies on silica and lung cancer, we had decided a priori that the relevant lag time between exposure and outcome would be 20 years. According to our profile likelihood calculation, the maximum likelihood was reached at 18 years, but other options from 13 to 20 years were not much worse. Hence our a priori selection was acceptable, but a slightly shorter lag time would have been even more justified. In situations in which there is no biological or other model for estimating the optimal length of the lag time, use of a Poisson regression in defining the likelihood profile is an option to be recommended.

Earlier studies on similar data also indicate that cross-sectional cohorts defined on the basis of a single self-reported census occupation give practically the same relative risk estimates for cancer as specific cohort studies utilizing complete occupational histories. Social class is also a satisfactory approximation for a whole range of lifestyle-related factors. The adjustment for social class often seems to give essentially the same results as the use of carefully collected data on specific lifestyle factors (2).

One purpose of this methodological exercise was to find a valid exposure metric for epidemiologic studies 
applying a job-exposure matrix. On the basis of theoretical considerations, we first constructed the best feasible metric from our exposure data and then simplified it step-by-step. The final conclusion as to the carcinogenic risk of silica would have been the same for most of the alternative exposure metrics. However, the result of our exercise may be specific for silica and lung cancer, and it certainly varies according to the distribution of the proportion of exposed and the mean level of exposure in occupations and the characteristics of the disease.

Adopting various threshold exposure levels in the modeling had some effect on the risk pattern. One may speculate whether the risk ratio series for the medium and high exposure categories, omitting all exposures below $0.20 \mathrm{mg} / \mathrm{m}^{3}$ (risk ratios 1.26 and 1.38 , respectively; metric 2 in table 5), is more correct than the respective series without the threshold (risk ratios 0.97 and 1.42). The threshold approach may provide some additional information on the shape of the exposure-response relationship.

The simple noncumulative proportion of exposed multiplied by the mean level of exposure-without a historical job exposure matrix - has been used in several earlier FINJEM-based analyses (16-18). Silica exposure has decreased over time in most occupations. If we would not have had information on historical exposure levels and would have been forced to estimate the cumulative exposure only on the basis of recent estimates, we would have had remarkably fewer cases in the highest exposure category and the risk estimate for the highest cumulative exposure category would have been lower $(1.31$; metric 2 in table 5$)$ than the one based on period-specific exposure estimates (1.42).

The estimated duration of exposure was not an important component of cumulative exposure in the risk estimation of lung cancer (metric 3 in table 5), because most of the lung cancer cases were diagnosed among men who had already ended their active worklife and therefore had the maximal exposure burden typical to their occupational category. An advantage of adding duration to the calculation of the proportion of exposed multiplied by the mean level of exposure is that it converts the units of the level $\left(\mathrm{mg} / \mathrm{m}^{3}\right)$ into units of the "dose" ( $\mathrm{mg} / \mathrm{m}^{3}$-years), which is potentially useful in quantitative risk assessment and standard setting. However, the values for cumulative exposure in our study should be interpreted with caution because the prevalence and level of exposure are estimated averages of entire occupational categories.

In our example, the case of silica exposure, the simplest possible metric for silica exposure that gave a risk pattern similar to that resulting from the preferred metric was the average cross-sectional exposure level among the exposed. On the other hand, the other basic component of each FINJEM stratum, the proportion of exposed persons, as the only exposure information did not give reasonable risk estimates. The explanation may be the different variability in that the level varied widely from 0.01 to $0.60 \mathrm{mg} / \mathrm{m}^{3}$ while the prevalence varied only a little, being close to $100 \%$ for most occupations (table 2). Hence the value for cumulative exposure in the case of silica was predominantly driven by the proportion of the exposed. However, for many other exposures the proportion of exposed may vary considerably, and therefore also the proportion of exposed should be included in the metric.

The stability in most occupations is so high that cross-sectional information on occupation corresponds rather well to the life-long occupational history (12). This is especially true for aged populations in which the turnover between occupations is low. However, there are several occupations with a high turnover rate. The exposure misclassification should be smaller, the higher the occupational stability, and therefore the stable occupations should show higher risk ratios than the unstable ones, provided that there is a causal relationship between exposure and cancer. We divided the population into two groups according to their occupational stability between two censuses (12). The more stable half of the occupations reached a similar risk ratio above 1.3 in the medium exposure levels of $0.1-9.9 \mathrm{mg} / \mathrm{m}^{3}$-years than was seen in the less stable occupations with estimated cumulative exposure of over $10 \mathrm{mg} / \mathrm{m}^{3}$-years (metrics 7-8 in table 5). None of the stable occupations involved so much silica exposure that its workers would have reached the cumulative exposure of $10 \mathrm{mg} / \mathrm{m}^{3}-$ years, and we could not therefore estimate the effect of exposure misclassification due to occupational instability in the highest exposure category. The alternative method for estimating occupational stability was to utilize occupation-specific information on average length of employment, which reduced the cumulative exposure values for unstable occupations and moved some of the observations towards lower exposure categories. This shift somewhat sharpened the risk ratio in the highest exposure category (RR 1.50).

For both of the cancer sites studied, it seemed to be important to adjust for social class. Very similar results were obtained by excluding all except the blue-collar workers (last model in table 3). Although the latter approach reduced the number of cases, the actual loss of study power was small because crystalline silica dust (as most industrial exposures) is rare among farmers and white-collar occupations.

Although smoking in today's Finland coincides with poorer socioeconomic status, in our modeling, smoking had an effect on lung cancer that was partly independent of social class. Our smoking data (1978-1991) are too recent in terms of causation of cancers diagnosed 
during 1971-1995, and adjusting for smoking will or may bias the result if the recent occupation-specific smoking habits do not correlate with those in earlier decades. Fortunately the time trends in smoking among Finnish men have shown a rather parallel decrease in most population subgroups, and therefore it is justified to use the smoking estimate of the FINJEM as a cofactor in models of smoking-related cancers for men. The parameter correlation between social class and smoking was rather high in some models. Therefore, the risk ratios calculated for social class and smoking in the multivariate model (table 4) should be interpreted with caution, although they apparently behave as expected a priori. While the estimates on occupational agents are mostly considered reliable, there is much room for improvement in the nonoccupational confounder data.

\section{Crystalline silica and cancer risk}

Because of the wide use of quartz-containing materials, workers are exposed to crystalline silica in a large variety of industries and occupation. In Finland the number of exposed workers is $60000-70000$, and $90 \%$ of them are men (FINJEM database). Respirable quartz levels exceeding $0.1 \mathrm{mg} / \mathrm{m}^{3}$ have frequently been found in metal and nonmetal mines, in granite quarrying and processing, crushed stone and related industries, in foundries, in the ceramics industry, in construction and in sandblasting operations. Red and gray granite probably have a stronger association with lung cancer than black granite (19).

We observed an increase in the risk ratio of lung cancer with increasing silica exposure, and the incorporation of sufficient lag time also produced a higher risk ratio. These findings can be interpreted as signs of causality. Our results also suggest a threshold in that the excess was mainly attributable to workers in occupations with an estimated cumulative exposure exceeding 10 $\mathrm{mg} / \mathrm{m}^{3}$-years or threshold exposure of at least $0.2 \mathrm{mg} /$ $\mathrm{m}^{3}$. In a pooled exposure-response analysis of 10 silica-exposed cohorts the relative risk of lung cancer was observed to increase when cumulative exposure exceeded $9 \mathrm{mg} / \mathrm{m}^{3}$-years (20). In this case our average exposure estimates give a risk coefficient similar to the individual level estimates of the pooled analysis. Overall, our results concerning increased lung cancer risk among workers exposed to silica dust are consistent with the IARC evaluation, which stated in 1997 that occupational exposure to crystalline silica is carcinogenic to humans (8).

No association between prostate cancer and silica exposure has been observed in earlier studies. This was also our finding. After adjustment for social class, the stantardized incidence ratio and risk ratio estimates were close to 1.0 in all categories, and there was no doseresponse with exposure or an increase with lag time. Our prior observations also indicate that FINJEM-based calculations are not likely to produce "false positive" results on exposure-cancer combinations when there should not be any association.

\section{Concluding remarks}

According to our study, crystalline silica dust increases the risk of lung cancer, which is in line with the findings of the majority of numerous earlier studies. We consider this an indication of the usefulness of the FINJEM approach in large national studies on cancer. Although some of the recommendations for requirements for exposure metrics and methodological aspects given in multivariate model construction may be specific to circumstances in Finland, or may be specific to silica dust and lung cancer, similar aspects, modified to local conditions, are worth following in other settings as well.

\section{Acknowledgments}

We are grateful to Dr Antti Uutela (Finnish Public Health Institute, Helsinki) for allowing us to use data on lifestyle factors, Ms Tuula Liukkonen (Finnish Institute for Occupational Health, Lappeenranta) for the exposure assessment of silica and asbestos, and Ms Luanne Siliämaa for the linguistic revision of the manuscript. The project was economically supported by the Finnish Cancer Foundation and the Work Environment Fund.

\section{References}

1. Lynge E, Thygesen L. Occupational cancer in Denmark: cancer incidence in the 1970 census population. Scand J Work Environ Health 1990;16 suppl 2.

2. Pukkala E. Cancer risk by social class and occupation: a survey of 109,000 cancer cases among Finns of working age. Basel: Karger; 1995. Contributions to Epidemiology and Biostatistics, vol 7 .

3. Andersen A, Barlow L, Engeland A, Kjærheim K, Lynge E, Pukkala E. Work-related cancer in the Nordic countries. Scand J Work Environ Health 1999;25 suppl 2.

4. Job exposure matrices. Southampton (United Kingdom): Medical Research Council, Environmental Epidemiology Unit; 1983. Scientific report, no 2.

5. Coughlin SS, Chiazze L. Job-exposure matrices in epidemiologic research and medical surveillance. In: Rempel D, editor. Occupational medicine. Philadelphia (PA): Hanley and Belfus; 1990. p 633-6. State of the art reviews, vol 5, no 3.

6. Special issue on industry-based job-exposure matrices. Int $\mathbf{J}$ Epidemiol 1993;22 suppl 2.

7. Kauppinen T, Toikkanen J, Pukkala E. From cross-tabulations to multipurpose exposure information systems: a new jobexposure matrix. Am J Ind Med 1998;33:409-17. 
8. International Agency for Research on Cancer (IARC). Some silicates, coal dust and para-aramid fibrils. Lyon: IARC; 1997. IARC monographs on the evaluation of carcinogenic risks to humans, vol 68.

9. Central Statistical Office of Finland. Population census 1970: occupation and social position. Helsinki: Central Statistical Office of Finland, 1974. Official Statistics of Finland VI C:104, vol IX.

10. Teppo L, Pukkala E, Lehtonen M. Data quality and quality control of a population-based cancer registry-experience in Finland. Acta Oncol 1994;33:365-9.

11. Helakorpi S, Patja K, Prättälä R, Aro A, Uutela A. Health behavior and health among Finnish adult population, spring 2002. Helsinki: National Public Health Institute; 2002. Publications of the National Public Health Institute, B12.

12. Kolari R. Occupational mobility in Finland 1975/1980/1985. Helsinki: Central Statistical Office of Finland; 1989. Studies 160.

13. Partanen T, Kauppinen T, Degerth R, Moneta G, Mearelli I, Ojajarvi A, et al. Pancreatic cancer and industrial branches and occupations in Finland. Am J Ind Med 1994;25:851-66.

14. Aitkin M. Profile likelihood. In: Armitage P, Colton T, editors. Encyclopedia of biostatistics. Chichester (United Kingdom): Wiley; 1999. p 3534-6.
15. Pukkala E, Weiderpass E. Socio-economic differences in incidence rates of cancers of the male genital organs in Finland, 1971-95. Int J Cancer 2002;102:643-8.

16. Weiderpass E, Pukkala E, Kauppinen T, Mutanen P, Paakkulainen $\mathrm{H}$, Vasama-Neuvonen K, et al. Breast cancer and occupational exposures in women in Finland. Am J Ind Med 1999;36:48-53.

17. Alguacil J, Kauppinen T, Porta M, Partanen T, Malats N, Kogevinas M, et al. Risk of pancreatic cancer and occupational exposures. Ann Occup Hyg 2000;44:391-403.

18. Wesseling C, Pukkala E, Neuvonen K, Kauppinen T, Boffetta $\mathrm{P}$, Partanen T. Cancer of the brain and nervous system and occupational exposures in Finnish women. J Occup Environ Med 2002;44:663-8.

19. Koskela R-S, Klockars M, Laurent H, Holopainen M. Silica dust exposure and lung cancer. Scand J Work Environ Health 1994;20:407-16.

20. Steenland K, Manntje A, Boffetta P, Stayner L, Attfield M, Chen J. Pooled exposure-response analyses and risk assessment for lung cancer in 10 cohorts of silica-exposed workers: and IARC multicentre study. Cancer Causes Control 2001;12:773-84.

Received for publication: 11 May 2004 